\title{
Development of user-friendly consumer and health professional resources for the antipsychotic clozapine: a New Zealand example
}

\author{
Nicola J Holmes, ${ }^{1}$ S Wayne Miles, ${ }^{2,3}$ Amanda J Wheeler ${ }^{4,5}$
}

\begin{abstract}
- Additional material is published online only. To view please visit the journal online (http://dx.doi.org/10.1136/ bmjinnov-2014-000006).

${ }^{1}$ Birmingham and Solihull Mental Health NHS Foundation Trust, Birmingham, UK ${ }^{2}$ Waitemata District Health Board, Auckland, New Zealand ${ }^{3}$ Department of Psychological Medicine, University of Auckland, Auckland,

New Zealand

${ }^{4}$ Griffith Health Institute, Griffith University, Brisbane, Australia ${ }^{5}$ Faculty of Medical and Health Sciences, University of Auckland, Auckland, New Zealand
\end{abstract}

\section{Correspondence to} Professor Amanda Wheeler, Griffith Health Institute, Griffith University, Brisbane 4131, Australia; a.wheeler@griffith.edu.au

Received 12 September 2014 Accepted 21 October 2014

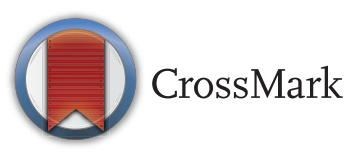

To cite: Holmes NJ, Miles SW, Wheeler AJ. BMJ Innov 2015;1:17-21.

\begin{abstract}
The antipsychotic clozapine has been shown to have superior efficacy to most other treatments for psychosis, especially where treatmentresistant schizophrenia has been diagnosed. Like all medications, clozapine has a number of wellrecognised adverse effects, some of which can be fatal. Evidence also suggests that those for whom clozapine is the most suitable treatment option do not receive it because the doctor or health system views the risks as too great to prescribe. The provision of guidelines including innovative consumer and general practitioner resources regarding the ways to most safely monitor and manage people who are exposed to clozapine has the potential to increase the safe use of the product and also to provide potential prescribers with more confidence in using it. This paper outlines both the process for development and the final package produced by a large metropolitan District Health Board service in Auckland, New Zealand. This material was aimed at non-specialist prescribers as well as consumers and their family/carers and other supports.
\end{abstract}

\section{INTRODUCTION}

Clozapine, the only antipsychotic recommended for treatment-resistant schizophrenia, has been available as a registered medicine in most English-speaking countries since the early 1990s. It was reintroduced after concerns related to fatal agranulocytosis had led to its withdrawal in 1975. A number of other lifethreatening adverse effects have been associated with clozapine, including myocarditis and cardiomyopathy, insulin resistance and diabetes, and constipation and bowel obstruction. Typically, owing to the risk of serious adverse effects, clozapine prescribing and dispensing is generally restricted to secondary care services. However, shared-care arrangements with primary healthcare services, that is, general practitioners (GPs) and community pharmacies, are increasing for those people who are stable and well established on this medication. Despite its superior efficacy and beneficial health outcomes, ${ }^{1}$ many studies show underutilisation, ${ }^{2-5}$ delayed access ${ }^{467}$ and a reluctance by psychiatrists to prescribe clozapine. ${ }^{8} 9$ In response to both safety and access concerns, clinical guidelines that recommend appropriate monitoring and management of mental and physical health for people initiated on clozapine are available in many countries. ${ }^{10-12}$

At a large public secondary care mental health service in Auckland, New Zealand, an organisation-wide evidence-based consensus-agreed clozapine best-practice guideline had been in place since 1999 . This guideline had been developed primarily for those clinicians involved in the prescribing, dispensing, administration and monitoring of clozapine within the secondary care mental health service. Regular reviews of the guideline addressed new evidence, as well as responding to outcomes of local case reviews and relevant Coronial enquiries throughout the country. Clozapine use had been reviewed regularly in the service as part of an audit and feedback cycle over 7 years between March 2000 and March 2007. ${ }^{6}$ 13-16 


\section{Call for the tool}

In late 2007, a New Zealand Coroner's report ${ }^{\mathrm{i}}$ regarding a clozapine-induced fatal blood dyscrasia that occurred 9 months after clozapine initiation highlighted that community-based people taking clozapine were being managed by both secondary care mental health services and GP services. It was felt that this conferred a potential for people to fall between the two services with respect to monitoring and management of adverse effects. The Coroner recommended that a "medication booklet to be carried by the patient" be developed that could act as an aide memoire for the consumer, their family and carers regarding the signs and symptoms of important adverse effects and how to respond to these, and provide advice to a practitioner (particularly nonregular practitioners and those in after-hours situations) about these adverse effects. Another Coroner's recommendation was that GPs be provided with "appropriate information about clozapine by specialist prescribers".

In response to this, the planned guideline review in 2008 included incorporating the Coroner's recommendations for developing appropriate, portable, user-friendly clozapine information resources for consumers and health practitioners who were not specialist clozapine prescribers. Other areas of concern identified by clinicians from within the organisation that were to be included in the review were

1. More detailed guidance on utility, rationale and interpretation of clozapine serum levels;

2. Initiation of clozapine in outpatient settings;

3. Monitoring and management of gastrointestinal adverse effects, particularly constipation;

4. Identification and management of significant and potentially fatal cardiovascular adverse effects, such as myocarditis and cardiomyopathy.

The work conducted around these areas is not presented here in detail, but is noted as it informed some content of the consumer and non-specialist health practitioner resources that were developed.

\section{METHOD}

\section{Development of the tool}

A multidisciplinary clozapine working group was established with different members leading the process for each of the four areas outlined above. The working group consulted with national and international specialists and the organisation's consumer advisor group as needed. The main tasks of the resource development workstream were to design and produce the portable resource for people taking clozapine and any healthcare professionals they came in contact with and also an information resource for GPs, as recommended by the Coroner's report.

\footnotetext{
'Coroner's findings in New Zealand are available free online for all completed cases opened after 1 July 2007. Cases opened prior to 30 June 2007 are available on request http://www.justice.govt.nz/courts/ coroners-court/publications/findings-recommendations-and-records.
}

An extensive search of the literature, Google and mental health pharmacy network groups in the UK, Australia and New Zealand was carried out to identify existing portable consumer resources and quick reference information for GPs about clozapine, the risk of adverse effects, and how to respond to these. Only one example of a portable consumer resource was identified from a mental health service in the UK, but this was more as a medical alert card than as a portable resource (personal correspondence Dr Dave Branford 30 December 2008). The card recorded the consumer's name, the prescriber's details, a warning that clozapine can cause neutropenia and to be on the alert for signs of infection, and emergency medical contact numbers at the Clozaril Patient Monitoring Service (CPMS). This UK mental health service added a sticker to the reverse side of this standard card provided by the CPMS, which included advice about restarting treatment, and the impact of smoking and contact details for the pharmacy supplying the clozapine.

A letter for GPs who would be working with people taking clozapine had already been developed for use by the Auckland mental health service prior to this most recent guideline update; however it required updating. Several other formal letter templates were identified, but these did not outline the benefits and risks of clozapine or give specific advice to the GP about the need for close haematological monitoring, the additional monitoring that should be carried out (by the GP) and in which circumstances, and when to contact the mental health team. Many of these letters had been developed by the pharmaceutical manufacturers of clozapine.

\section{RESULTS}

Three draft resources were developed over an 18-month period

1. A double-sided wallet leaflet that folded up to bankcard size to be carried by consumers (one side detailing information for consumers and the other side for health professionals) (see online supplementary appendix A);

2. A two-page letter for GPs alerting them that someone under their care had been started on clozapine and summarising important related information (see online supplementary appendix B);

3. A one-page quick reference sheet available on the organisation's external website (see online supplementary appendix C).

The three resources were designed to supplement the very detailed information about clozapine that is included in the manufacturers' registration documentation available on the Medsafe ${ }^{\mathrm{ii}}$ website (http://www. medsafe.govt.nz).

\footnotetext{
${ }^{i i}$ Medsafe is the New Zealand Medicines and Medical Devices Safety Authority. It is a business unit of the Ministry of Health and is the authority responsible for the regulation of therapeutic products in New Zealand.
} 
The consumer resource was developed with significant input from the organisation's consumer advisor group. Space was included to document personal details and the mental health professionals working with the person carrying the card. This document could be folded to the same size as a bankcard to fit in a wallet or purse (figure 1). Two consumer advisors then piloted the prototype with eight people taking clozapine, and minor changes were made to the wording based on their feedback. The information letter for GPs was piloted with three GPs who had consumers taking clozapine in their practice, and again minor amendments were made due to their feedback.

While working towards final versions of the consumer and GP resources, it became apparent that there needed to be a more detailed summary resource that could easily be accessed by people taking clozapine, their families/carers, and healthcare professionals working with people taking clozapine. This quick reference sheet was developed to be accessible on the internal intranet and external organisation website, and it is referred to in both the GP resource and the wallet card as an additional resource.
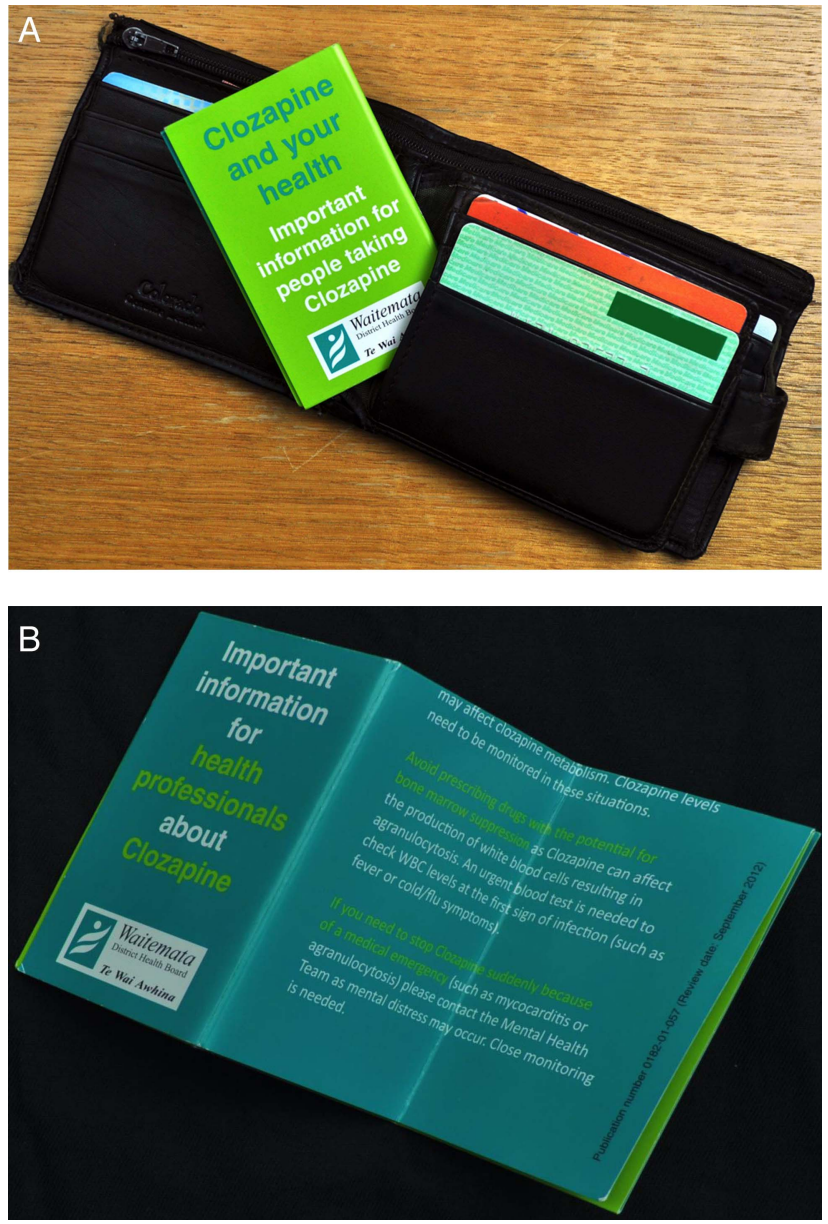

Figure 1 ( $A$ and $B$ ) Portable information resource for consumers and health professionals (wallet card).
Finally, the resources were presented widely across the organisation at staff and professional meetings as part of the consultation process for the updated guideline. Overall feedback from staff, consumers and GPs was positive. Suggested amendments were incorporated where relevant, and roadblocks solved where possible.

All three resources were incorporated into the final version of the guideline, with signposting on the wallet card directing staff to order bulk supplies of the printed and folded cards from the pharmacy department (see online supplementary Appendices A-C). The GP letter and quick reference sheet were made available on the internal intranet, and additionally the reference sheet was available on the external website to facilitate ease of access (http://www.waitematadhb. govt.nz/HealthProfessionals/Medicinesresources.aspx).

Prior to the final sign-off and publication, the guideline was reviewed and approved by the organisation's Drug and Therapeutics Committee.

\section{DISCUSSION}

\section{Strategies for innovation}

The strategies used to update the guideline to support evidence-based practice in a consensus-agreed manner were undertaken to maximise awareness of and adherence with the best-practice recommendations and have been discussed previously. With respect to dissemination, the updated guideline was emailed electronically to all mental health clinical staff, discussed with medical staff employed at the time, and then embedded into induction training for new staff starting with the organisation. This occurred across inpatient, community and forensic services. Copies of the consumer wallet card, GP letter and summary resources were placed in the organisation's consumer advice folders available in all clinical areas. In addition, a specific initiative took place whereby care coordinators were tasked with ensuring that all community-based consumers taking clozapine received a wallet card.

An informal clozapine initiation checklist (aimed specifically at junior medical staff but available for any staff to use) had previously been in place within the organisation; however, it had not explicitly been part of the best practice guideline. In order to facilitate clarity regarding the monitoring and steps required prior to starting clozapine (especially for junior medical staff not necessarily as familiar with this medication as more senior medical staff), this checklist was reviewed and updated and integrated within the guideline (see online supplementary appendix D). Junior medical staff were consulted on the checklist and it was piloted on an inpatient forensic unit prior to being finalised and implemented; feedback regarding the clarity and ease of use of the document was positive. The checklist was also made accessible separately on the internal intranet to facilitate ease of access. 
In the final stages of guideline dissemination, a subsequent Coroner's report (following a cardiac adverse event) recommended that the updated guideline be available to other health services throughout the country, to Medsafe and to the Health Quality and Safety Commission. ${ }^{\mathrm{iii}}$ More specifically, the Coroner recommended that the Commission:

Assists in providing national leadership to ensure that the clozapine guidelines of all District Health Boards are aligned with international best practice standards and consistent across the country;

Identifies a process for developing national best practice guidelines for the prescription and monitoring of medications such as clozapine for which practitioners throughout New Zealand require particular guidance.

The guideline developers were aware that, as the guideline had been developed for local application, it would not meet international criteria set by the Appraisal of Guidelines for Research and Evaluation (AGREE) Trust (http://www.agreetrust.org). It was also acknowledged that a guideline that was consensusagreed in one setting would need to be assessed by another organisation independently to elucidate utility, and agreement from clinicians; otherwise, adherence could be compromised. To help clarify these considerations for organisations, the guideline was shared with a cover note that highlighted these issues and also provided a summary of the process followed and staff involved with respect to the expert consensus groups and associated consultees.

\section{Lessons and messages}

The guideline took an extended amount of time to update and disseminate (approximately 2.5 years). This was partially due to (1) the logistical difficulties associated with gathering all the relevant expertise together for the working group, and especially for consultation outside the organisation, plus, (2) the work was undertaken by busy health practitioners who had to incorporate this work into their current workload with no backfill. Ideally, ring-fenced time would have been available to expedite the process. The extended nature of the review meant that some enlisted staff moved on to other settings or organisations during the review process; replacements had to be enlisted and brought up to speed prior to being able to contribute meaningfully.

Another complication was a succession of relevant evidence and reports being published during guideline development. It was a challenge to incorporate newly available information as and when it became available,

\footnotetext{
iiiThe Health Quality \& Safety Commission (http://www.hqsc.govt.nz) was established in November 2010 to ensure that all New Zealanders receive the best health care and disability care within available resources and with an expectation from the Government that it would lead quality and safety improvements in the health sector.
}

and also to make a decision on when to stop developing the guideline so that it could be signed off and implemented.

The use of a widely consultative, consensus-agreed and collaborative approach with clinicians and other health professionals throughout the guideline and resource review and development process, alongside inclusion within the guideline of content requested by staff, facilitated implementation of the finished guideline, as many staff were already aware of and familiar with the content.

Informal feedback from clinical pharmacists and cardiology services suggested that the guideline was being followed throughout inpatient and community mental health services within the organisation. Informal feedback from consumers, mental health clinicians and other staff indicated that they felt the guideline contents, especially the associated resources, were useful.

For guidelines and best practice summaries to have the most impact on clinical decision-making, it is crucial that they remain current and that essential details are readily available at both the time and site of that decision. This has historically not been easy to achieve, partly due to the workload involved with updating guidelines in the absence of specific resourcing for this, and also due to limitations in the logistics of information technology. The organisation has developed CeDSS (Clinical electronic Decision Support System), which provides practitioners with key information at the bedside. It has yet to be extended to outside hospital use. When that is achieved, then this clozapine-related material will be easily and rapidly accessed. The material on this site is regularly reviewed by expert content holders and this will ensure that information is regularly updated.

The guideline was due for review in mid-2013. Many of the original working group members have left the organisation. Efforts have been made to maintain relevant expertise and knowledge base within the organisation's mental health pharmacy services to facilitate seamless progress of further guideline updates.

Ideally, the implementation of the guideline would improve the safe and quality use of clozapine within the organisation. This is difficult to state with certainty, given the lack of available formal evidence regarding this, as a post-implementation audit has not occurred. Post-implementation audits are important to identify adherence with guideline recommendations and identify strategies to address any areas of poor adherence. The significant adverse effects discussed, while being potentially fatal, are rare, and as concluded in the Coroner's reports and evidence from the literature ${ }^{17-19}$ examining the utility of the mandatory full blood count monitoring, even good monitoring does not guarantee good outcomes. If people with difficult-to-treat schizophrenia are to have the best opportunity to minimise the effects of that illness and thereby maximise their recovery of roles and function, then it is important that they have 
access to well-informed clinicians who have welldeveloped systems for safe and reliable monitoring of mental and physical health. The described set of tools to inform consumers and their families, as well as clinicians at the secondary and primary levels, meet these requirements. Future audit of clozapine use at the organisation will allow evaluation of the impact of the set of tools on enhancing quality use of clozapine.

Acknowledgements The authors would like to thank all the members of the organisation's clozapine working group, the organisation's consumer advisor team, the many other clinicians and consumers who contributed their expertise in the consultation and piloting process, and the administrative staff who assisted in the development of resources and document formatting.

Contributors Each author contributed to the guideline review, consultation and dissemination. NJH led the serum level monitoring work and the dissemination process. SWM was a member of the working group specifically reviewing cardiac monitoring. AJW led the overall review process and development of the resources. Each author contributed to and approved the final version of the manuscript.

\section{Competing interests None.}

Provenance and peer review Not commissioned; externally peer reviewed.

\section{REFERENCES}

1 Tiihonen J, Lönnqvist J, Wahlbeck K, et al. 11-Year follow-up of mortality in patients with schizophrenia: a population-based cohort study (FIN11 study). Lancet 2009;374:620-7.

2 Downs J, Zinkler M. Clozapine: national review of postcode prescribing. Psychiatr Bull 2007;31:384-7.

3 Sernyak MJ, Rosenheck RA. Antipsychotic use in the treatment of outpatients with schizophrenia in the VA from fiscal years 1999 to 2006. Psychiatr Serv 2008;59:567-9.

4 Nielsen J, Røge R, Schjerning O, et al. Geographical and temporal variations in clozapine prescription for schizophrenia. Eur Psychopharmacol 2012;22:818-24.

5 Hermes E, Rosenheck R. Choice of randomization to clozapine versus other second generation antipsychotics in the CATIE schizophrenia trial. J Psychopharmacol 2012;26:1194-200.

6 Wheeler AJ. Treatment pathway and patterns of clozapine prescribing for schizophrenia in New Zealand. Ann Pharmacother 2008;42:852-60.
7 Taylor DM, Young C, Paton C. Prior antipsychotic prescribing in patients currently receiving clozapine: a case note review. J Clin Psychiatry 2003;64:30-4.

8 Nielsen J, Dahm M, Lublin H, et al. Psychiatrists attitude towards and knowledge of clozapine treatment. J Psychopharmacol 2010;24:965-71.

9 Cirulli G. Clozapine prescribing in adolescent psychiatry: survey of prescribing practice in in-patient units. Psychiatr Bull 2005;29:377-80.

10 National Collaborating Centre for Mental Health. Schizophrenia: core interventions in the treatment and management of schizophrenia in primary and secondary care. Clinical Guideline 82. Secondary Schizophrenia: core interventions in the treatment and management of schizophrenia in primary and secondary care. Clinical Guideline 82. 2009. http://www.nice.org.uk/CG82

11 Barnes TR. Evidence-based guidelines for the pharmacological treatment of schizophrenia: recommendations from the British Association for Psychopharmacology. J Psychopharmacol 2011;25:567-620.

12 Royal Australian and New Zealand College of Psychiatrists Clinical Practice Guidelines Team for Depression. Australian and New Zealand clinical practice guidelines for the treatment of depression. Aust N Z J Psychiatry 2004;38:389-407.

13 Wheeler A, Humberstone V, Robinson G. Outcomes for schizophrenia patients with clozapine treatment: how good does it get? J Psychopharmacol 2009;23:957-65.

14 Wheeler AJ, Humberstone V, Robinson E, et al. Impact of audit and feedback on antipsychotic prescribing in schizophrenia. J Eval Clin Pract 2009;15:441-50.

15 Wheeler A. Explicit versus implicit review to explore combination antipsychotic prescribing. J Eval Clin Pract 2009;15:685-91.

16 Wheeler AJ, Feetam C, Harrison J. Pathway to clozapine use: a comparison between a patient cohort from New Zealand and a cohort from the United Kingdom. Clin Drug Invest 2014;34: 203-11.

17 Alvir J, Lieberman J, Safferman A, et al. Clozapine-induced agranulocytosis-incidence and risk factors in the United States. N Engl J Med 1993;329:162-7.

18 Honigfeld G. The clozapine national registry system: forty years of risk management. J Clin Psychiatry 1996;14:29-32.

19 Krupp P, Barnes P. Leponex-associated granulocytopenia: a review of the situation. Psychopharmacol 1989;99:118-21. 